\title{
Identification of a Bacillus Thuringiensis Cry8Ea3 to Xin-Binding Alkaline Phosphatase from Holotrichiaparallela Wei WANG ${ }^{1}$, Dan $Z \mathrm{HAO}^{1}$, Wei GUO ${ }^{2, a, *}$, Xiao-ping YAN ${ }^{1}$, Ya-kun ZHANG ${ }^{1}$, Kun-li ZHAO', Yu-jie GAO and Xiao-yun WANG ${ }^{3}$ ${ }^{1}$ College of Plant Protection, Agricultural University of Hebei, Baoding, Hebei, China \\ ${ }^{2}$ College of Plant Science and Technology, Beijing University of Agriculture, Beijing, China \\ ${ }^{3}$ College of Agriculture, Northeast Agriculture University, Harbin, Heilongjing, China College of Plant Science and Technology, Beijing University of Agriculture, Beijing, China; \\ a1787421502@qq.com; \\ ${ }^{*}$ Corresponding author;
}

Keywords: Holotriciaparallela, Alkaline phosphatase, Ligand blot, qRT-PCR.

\begin{abstract}
In order to further study the Insecticidal Mechanism of Bt protein against Holotriciaparallela. In the study, According to the results of midgut transcriptome sequencing and RACE-PCR, the full-length hpalp gene was cloned. Sequencing analysis showed that the open reading frame of hpalp (GenBank accession NO. KY922835) was 1605 bp long, encoding 534 amino acid residues. The predicted molecular weight and isoelectric point of HpALP were $59 \mathrm{kDa}$ and 5.18, respectively. Analysis of HpALP signal peptide with 21 amino acids, GPI anchor point is located in the C-terminal $\mathrm{D}^{514}$, and has two $\mathrm{N}$-glycosylation sites: $\mathrm{N}^{100}, \mathrm{~N}^{296}$. The results of Ligand blot showed that the binding of HpALP with Cry8Ea3 toxin. Transcriptional analysis of hpalp in different tissues of $H$. parallela larvae was performed by qRT-PCR, which revealed that the hpalp was primarly expressed higher in midgut, but lower in the foregut.
\end{abstract}

\section{Introduction}

Holotriciaparallela belongs to the order Coleoptera, family Scarabeidae, and its larvae are commonly recognized as the most significant domestic underground pest affecting peanut, sweet potato, and potato which caused substantial reductions in crop yields and huge economic losses[1,2]. Bacillus thuringiensis (Bt) toxin Cry8Ea had specific activity against Holotriciaparallela larvae[3].

Research showed that the mechanism of insecticidal effect of $\mathrm{Bt}$ has been demonstrated to be related to brush border membrane vesicles (BBMV) receptor protein in the insect midgut epithelial cells[4,5]. There were two potential modes to explain the underlying mechanism of toxicity of Cry proteins. In the pore formation [6,7], the toxin-receptor interaction would promote toxin oligomerization and membrane-pore formation, resulting in midgut cell lysis and the eventual death of the target larvae. In the signal transduction model[8], the Cry toxins binding cadherin receptors caused a intracellular signal transduction, $\mathrm{G}$ protein and adenosine cyclase are activated, elevated cyclic adenosine monophosphate (cAMP), and protease A is activated, eventually leading to cells death. A number of receptors have been described for Cry toxins, including cadherin-like[9,10], aminopeptidase N (APN)[11,12], 
alkaline phosphatase (ALP) [13,14], ABC transporter (ABCC2) [15].

In this work, the cDNA of the full-length $H$. parallela alp (hpalp) gene was cloned from larvae midguts for the first time, and the HpALP protein was separated from Escherichia coli. To test the binding ability of the protein with the coleopteran-specific toxin Cry8Ea3, ligand blot experiments were carried out. Furthermore, we used quantitative reverse transcription-polymerase chain reaction (qRT-PCR) to determine the transcriptional level of the hpalp gene in different larval tissues of $H$. parallela.

\section{Materials and Methods}

\section{Insects}

H. parallela were collected from the field in Baoding, China, and the adults were reared with fresh Ulmus pumila elm tree leaves in the rearing chamber. The larvae were reared with artificial feed.

\section{Preparation of Cry Toxin}

BTGWL, Cry8Ea3 (GenBank No. KC855216.1) engineering strains were cultured in 1/2LB medium supplemented with ampicillin and erythromycin at $30 \pm 1^{\circ} \mathrm{C}$ until complete autolysis and spore crystal mixtures were prepared. The cry8Ea3 crystal proteins were prepared by differential centrifugation. Cry8Ea3 protoxin was activated by trpsin and analyed by SDS-PAGE.

\section{Cloning of the Hpalp Gene}

Total RNA was extracted $15 \mathrm{mg}$ of fresh midguts of third-instar $H$. parallela larvae using the RNAprep pure Tissue Kit (Tiangen, Beijing, China). The first-strand cDNA was synthesized according to the GoScript ${ }^{\mathrm{TM}}$ Reverse Transcription System (Promega, Madison, WI, USA). Simultaneously, 5'-RACE primer was designed according to the results of $H$. parallela midgut transcriptome sequencing and amplified by SMARTER RACE cDNA Amplification Kit (Clontech, Mountain View, CA, USA). The PCR program was as follows: 5 cycles of $94^{\circ} \mathrm{C}$ for $30 \mathrm{~s} ; 5$ cycles of $94^{\circ} \mathrm{C}$ for $30 \mathrm{~s}, 70^{\circ} \mathrm{C}$ for $30 \mathrm{~s}$, and $72^{\circ} \mathrm{C}$ for $3 \mathrm{~min}$; and 27 cycles of $94^{\circ} \mathrm{C}$ for $30 \mathrm{~s}, 68^{\circ} \mathrm{C}$ for $30 \mathrm{~s}$, and $72^{\circ} \mathrm{C}$ for 3 min. Next, using Primer 5.0 designerate full length primers (alp-F/alp/R). The PCR program was as follows: one cycle of $94^{\circ} \mathrm{C}$ for $4 \mathrm{~min}$; 30 cycles of $94^{\circ} \mathrm{C}$ for $45 \mathrm{~s}, 67^{\circ} \mathrm{C}$ for $45 \mathrm{~s}$, and $72^{\circ} \mathrm{C}$ for $1 \mathrm{~min}$ and $40 \mathrm{~s}$; and a final cycle at $72^{\circ} \mathrm{C}$ for $10 \mathrm{~min}$.

\section{Expression of HpVAA Protein in E. Coli BL21}

The open reading frame of hpalp was amplified, purified with Universal DNA Purification Kit (TIANGEN, Beijing, China), and cloned into pET21b vector via restriction enzymes digestion ( Sal I and Not I).

The strain BL21 (pET21b-hpalp) was cultured in Luria-Bertani medium at $37^{\circ} \mathrm{C}$, and induced by isopropyl- $\beta$-D-1-thiogalactopyranoside for $4 \mathrm{~h}$ and $8 \mathrm{~h}$; BL21(pET21b) served as the negative control. HpALP protein was loaded onto $12 \%$ SDS-PAGE and then transferred to polyvinylidene fluoride membranes to determine its expression. In brief, the membrane with the transferred proteins was blocked for $1 \mathrm{~h}$ with $1 \%$ bovine serum albumin at room temperature. After blocking the membrane was incubated with anti-His (1:5000 in Tris-buffered saline [TBS]) mouse antibodies (Boster, Wuhan, China) for $1 \mathrm{~h}$, followed by washing with TBS four times for $5 \mathrm{~min}$, and then with 
Tween (TBS-T) washing once for $5 \mathrm{~min}$. The membrane was subsequently incubated with second antibodies (goat anti-mouse antibodies, 1:10000 in TBS) for $1 \mathrm{~h}$, and the same washing steps were applied as described above. Detection was performed with Nitrotetrazolium Blue chloride (NBT) and 5-Bromo-4-Chloro-3-Indolyl Phosphate (BCIP).

\section{Ligand Blot Analysis of HpVAA Protein}

After separating with $12 \%$ SDS-PAGE, HpALP protein was transferred onto a polyvinylidene fluoride membrane, and incubated in blocking buffer, $1 \%$ bovine serum albumin in TBS, for $1 \mathrm{~h}$. The blocked membrane was then incubated with 20 $\mu \mathrm{g} / \mathrm{mL}$ Cry8Ea3 toxin proteins, activated by trypsin in blocking buffer, and washed with TBS and TBS-T. The blocked membrane was incubated with Cry8Ea3 primary antibodies (rabbit polyclonal antibody, 1:5000 in TBS) for $1 \mathrm{~h}$ and then conjuncted with anti-rabbit secondary antibody for $1 \mathrm{~h}$. The immunoblots were developed with NBT and BCIP.

\section{qRT-PCR}

Total RNA was traditionally separated from the midgut, foregut, hindgut, Malpighian tubes, fat body, peritrophic membrane, and egg of $H$. parallela. cDNA was synthesized and primers were designed as described above. The cDNA was amplified by qRT-PCR with specific primers (CFX96 Touch $^{\mathrm{TM}}$ Real-Time PCR System, BIO-RAD, Germany). PCR amplification conditions were as follows: one cycle of $95^{\circ} \mathrm{C}$ for $30 \mathrm{~s}, 39$ cycles of $95^{\circ} \mathrm{C}$ for $5 \mathrm{~s}, 56^{\circ} \mathrm{C}$ for $30 \mathrm{~s}$, and $72^{\circ} \mathrm{C}$ for $30 \mathrm{~s}$, one cycle of $95^{\circ} \mathrm{C}$ for $1 \mathrm{~min}$, one cycle of $50^{\circ} \mathrm{C}$ for $1 \mathrm{~min}$, one cycle of $65^{\circ} \mathrm{C}$ for $5 \mathrm{~s}$. The relative quantities of hpalp transcripts were assessed using the $2^{-} \Delta^{\mathrm{Ct}}$ method and normalized with $\beta$-actin.

\section{Results}

\section{Cloning and Analysis of the Hpalp Gene of H. Parallela}

The full-length sequence of hpvaa cDNA was successfully obtained by RACE-PCR (Table 1A). The full-length hpalp gene was cloned. Sequencing analysis showed that the open reading frame of hpalp (GenBank accession NO. KY922835) was 1605 bp long encoding 534 amino acids with a predicted molecular weight and isoelectric point of HpALP were $59 \mathrm{kDa}$ and 5.18, respectively. The SignaIP 4.1 Server on-line analysis of HpALP signal peptide with 21 amino acids; Big-PI Predictor analysis of GPI anchor point is located in the C-terminal $\mathrm{D}^{514}$; NetOGlyc 3.1 Server online analysis found no O-glycosylation site; Server NetNGlyc1.0 online prediction has two N-glycosylation sites: $\mathrm{N}^{100}, \mathrm{~N}^{296}$ (Figure 1A). Additionally, using MEGA 6.0 and the neighbor-joining (NJ) construct phylogenetic tree, HpALP was closely related to Nicrophorus vespilloidese ALP (GenBank accession NO. XP008200193.1) (Figure 1B). Ag, Anoplophora glabripennis; Tc, Tribolium castaneum; Ap, Agrilus planipennis; At, Aethina tumida; Dp, Dendroctonus ponderosae; Nv, Nicrophorus vespilloides. SWISS-MODLE on line was used to 3D-modeled structure of HpALP (Figure 1C). 
Table 1. Summary of primers used in the study

\begin{tabular}{cccc}
\cline { 2 - 3 } Primer & Orientation & Primer DNA sequence \\
\cline { 2 - 4 } 5-RACE & 5'RACE & CTTCCGAATAGACGAGTTGATGGGCG \\
Full-F & Forward & gcgtcgacATGAACGCGAAGTGTGTG \\
Full-R & Reverse & ttgcggccgccGTTTCTTCCGTCATTGGA \\
qPCR/F & Forward & CCATCTAGACGCTGAAGATAAC \\
qPCR/R & Reverse & TGTGCTCTAGTTTCGTGATG \\
Actin/F & Forward & ATGTTGCCATCCAAGCTGTA \\
Actin/R & Reverse & CCAAACGCAAAATAGCATGA \\
\hline
\end{tabular}

1 MNAKCVLVLI SVIGLVVTIQ ARPENFANSY DDGHMHPNPV KRSINTKTSK INAEEYNSKF WIDQAQKVLN

71 AKLLEKPNTN IAKNVIMFLG DGLSIPTIKA SRLYLGQLHN ESGEETILSF EKFPYVGLSK TYCVDTQVPD

141 SACTATAYLS GVKNNYHTIG VTAAVQYQDC DASLNEENHV YSIAQWSQLK NKKTGIVTTT RITHASPAGA

211 YANTADRDFE SDADTPDSKC KDIAHQLVYS EVGKNLNVVL GGGRREFLPN TVTDEGGQRG RRLDGENLIE

281 AWLKSKQGHK AQYVWNRTEL ENVAGDTEYL LGLFEDNHCK YHLDAEDNDP SLTEMTEKAI ELLASAENGY

351 FLFVEGGRID HAHHETRAQK ALDETVEFHK AIERAVELTN KEDTLIVVTA DHAHTMSVSG YAERGNDILG

421 ICDKADDELP YMTLSYANGP GYRASVGGRR TDVTNDNTHD KNYRFPVIAP LEDETHGGDD VSIYSQGPWS

491 HLLTGVIEQN VIPHIMAYAS CVGDGLKICD QELERIKKSN DGRN*

B

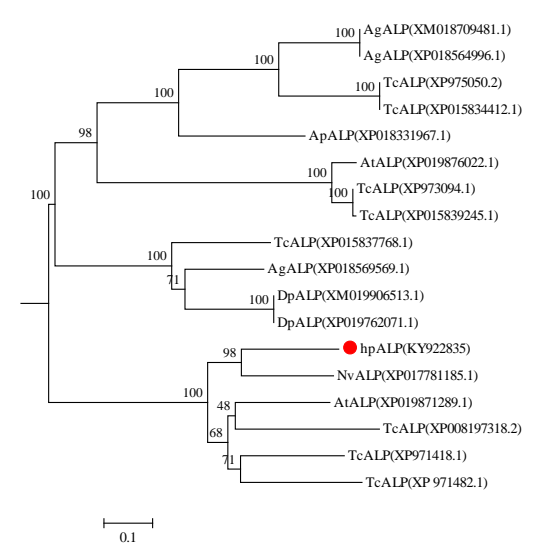

$\mathrm{C}$

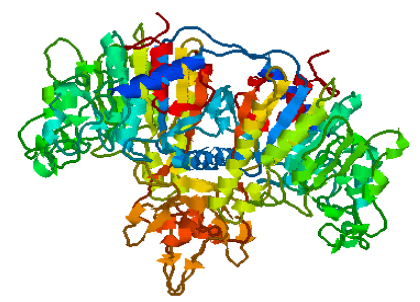

Figure 1. Gene sequence analysis of ALP from H. parallela. Panel A, The Amino acid sequence of ALP was deduced from $H$. parallela cloned of alp gene (GenBank accession NO. KY922835). Signal peptide is underlined with a double line. N-linked glycosylation sites by a solid triangle. GPI-anchor site by font-bold. Panel B, Phylogentic tree based on amino acid sequences of HpALP from H. parallela and other known insects. HpALP was closely related to Nicrophorus vespilloidese ALP (GenBank accession

NO. XP008200193.1). Ag, Anoplophora glabripennis; Tc, Tribolium castaneum; Ap, Agrilus planipennis; At, Aethina tumida; Dp, Dendroctonus ponderosae; Nv, Nicrophorus vespilloides. Panel C, 3D-modeled structures of HpALP.

\section{Expression of pET21b-Hpalp in E. coli}

About $69 \mathrm{kDa}$ band was visible on the gel of SDS-PAGE (Figure 2A), which was consistent with the predicted molecular weight. The recombinant protein existed in the form of an inclusion body. Western blot analysis demonstrated a clear signal with a band appearing at $69 \mathrm{kDa}$ (Figure 2B). 


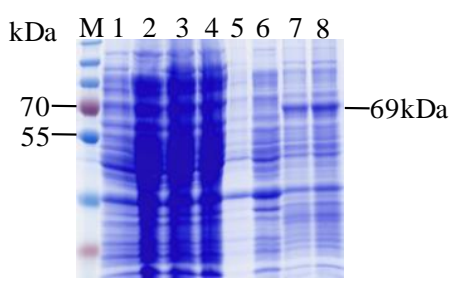

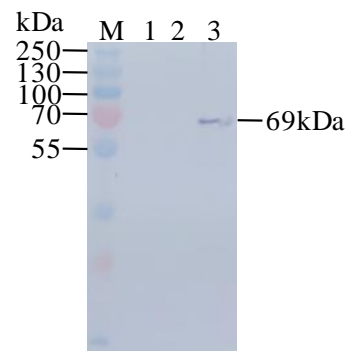

Figure 2. Identification and analysis of a recombinant HpALP protein. Panel A, Expression of the recombinant HpALP protein analyzed by SDS-PAGE in E. coli. M: Protein Marker; lanes 1-4: supernatant; lanes 5-8: precipitate. Lanes 1, 5: BL21(pET21b); 2, 6: BL21(pET21b-hpalp), not induced; 3, 4, 7, and 8: BL21(pET21b-hpalp) induced for $4 \mathrm{~h}$ and $8 \mathrm{~h}$, respectively. Panel B, Western bot analysis of the recombinant expression of HpALP in E. coli. M: Protein Marker; lane 1: BL21(pET21b); 2 : BL21(pET21b-hpalp), not induced; 3: BL21(pET21b-hpalp), induced.

\section{Ligand Blot of HpALP Protein in E. coli BL21}

Ligand blot of Cry8Ea3 toxins, as well as immune-blot for HpALP protein are shown in. The Cry8Ea3 ligand blot revealed strong binding to a protein of $69 \mathrm{kDa}$ (Figure 3).

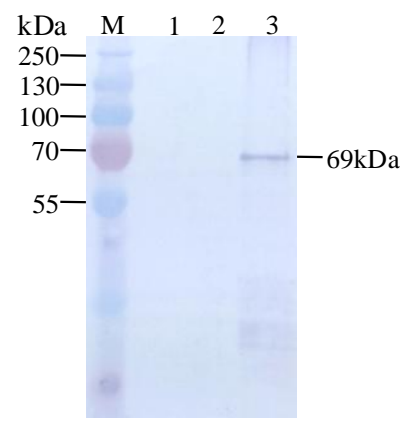

Figure 3. Ligand blot analysis of recombinant expression of HpALP in E. coli M: Protein Marker; lane 1: BL21(Pet21b); 2: BL21(pET21b-hpalp), not induced; 3: BL21(pET21b-hpalp), induced.

\section{Transcriptional Analysis of Hpalp in Different Larval Tissues of $\boldsymbol{H}$. Parallela}

Transcriptional analysis of hpalp in different tissues of $H$. parallela larvae was performed by qRT-PCR, which revealed that the hpalp was primarly expressed higher in midgut, but lower in the foregut (Figure 4).

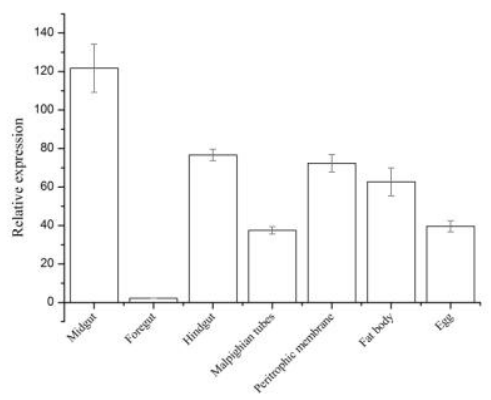

Figure 4. Relative expression level of hpalp in different tissue in the third larvae of H. parallela 


\section{Discussion}

In the $S$. exigua midgut, ALP acts as Cry1Ac binding protein ${ }^{[16]}$. ALP1 silenced Aedes aegypti larvae showed tolerance to both Cry4Ba and Cry11Aa, but not tolerance to Cry4Aa. The result shows that ALP1 was a functional receptor that plays an important role in the toxicity of the Cry4Ba and Cry11Aa proteins[17]. Immunolocalization results showed that Cry4Ba is able to bind to only $\mathrm{Sf9}$ cells-expressing AaALP. Moreover, these cells were shown to undergo cell lysis in the presence of trypsin-treated toxin. Finally, trypan blue exclusion assay also demonstrated an increase in cell death in recombinant cells treated with Cry4Ba. Overall results indicated that AaALP protein was responsible for mediating Cry4Ba toxicity against Sf9 cells, suggesting its role as a receptor for Cry4Ba toxin in A. aegypti larvae[18]. Further studied have found that the binding site is located on the domain II loop 2 of the Cry4Ba protein[17]. In the present study, ligand blot assay showed binding of the HpALP with Cry8Ea3 toxin.

In the S. exigua, ALP2 was expressed in the whole larval period, the lowest expression levels in the 1st instar larvae, and the highest expression in the 4th instar larvae[16]. However, The ALP could be expressed in the 1st, 2nd, 3rd larval period, and there were significant differences among different instars. The lowest and highest expression levels appeared in the 1st and 3rd instar larvae, and no expression in 4th and 5th instar larvae[19], in the Manduca sexta. In the work, transcriptional analysis of hpalp in different tissues of $H$. parallela larvae, which revealed that the hpalp was primarly expressed higher in midgut, but lower in the foregut.

\section{Acknowledgement}

This work was supported in part by the earmarked fund for Modern Agro-industry Technology Research System (CARS-14) and the Natural Science Foundation of Hebei Province (C2016204082).

\section{References}

[1] Yu H, Zhang J, Huang D et al. Characterization of Bacillus thuringiensis strain Bt185 toxic to the Asian cockchafer: Holotrichia parallela, Curr. Microbiol. 53 (2006) 13-17.

[2] Shu C, Yu H, Wang R, et al. Characterization of two novel Cry8 proteins from Bacillus thuringiensis strain BT185, Current. Microbiol. 58 (2009b): 389-392.

[3] Shu C, Yan G, Wang R, et al. Characterization of a novel Cry8 protein specific to Melolonthidae pests: Holotrichia oblita and Holotrichia parallela, Appl. Microbio. Biotechnol. 84 (2009a ) 701-707.

[4] Bravo A, Hendrickx S, Jansens S, et al. Immunocytochemical analysis of specific binding of Bacillus thuringiensis insecticidal crystal proteins to lepidopteran and coleopteran midgut membranes, J. Invertebr. Pathol. 60 (1992) 247-254.

[5] Soberon M, Gill S, Bravo A. Signaling versus punching hole: How do Bacillus thuringiensis toxins kill insect midgut cells? Cell. Mol. Life. Sci. 66 (2009) 1337-13349. 
[6] Pardo-López L, Gómez I, Munoz-Garay C, et al. Structural and functional analysis of the pre-pore and membrane-inserted pore of Cry1Ab toxin, J. Invertebr. Pathol. 92 (2006a) 172-177.

[7] Pardo-López L, Soberón M, Bravo A. Bacillus thuringiensis insecticidal three-domain Cry toxins: mode of action, insect resistance and consequences for crop protection, FEMS. Microbiol. 37 (2013) 3-22.

[8] Zhang X, Candas M, Griko NB, et al. Amechanism of cell death involving an adenylyl Cyclase/PKA signaling pathway is induced by the Cry1 Ab toxin of Bacillus thuringiensis, Proc. Natl. Acad. Sci. USA. 103 (2006) 989-9902.

[9] Fabrick J, Oppert C, Lorenzen MD, et al. A novel Tenebrio molitor cadherin is a functional receptor for Bacillus thuringiensis Cry3Aa toxin, J. Biol. Chem. 284 (2009) 18401-18410.

[10]Zúñiga-Navarrete F, Gómez I, Peña G, et al. Identification of Bacillus thuringiensis Cry3Aa toxin domain II loop 1 as the binding site of Tenebrio molitor cadherin repeat CR12, Insect. Biochem. Mol. Bio. 59 (2015) 50-57.

[11] Aratee A, Kusol P, Gerd K, et al. Two specific membrane-bound aminopeptidase $\mathrm{N}$ isoforms from Aedes aegypti larvae serve as functional receptors for the Bacillus thuringiensis Cry4Ba toxin implicating counterpart specificity, Biochem. Biophys. Res. Commun. 461 (2015) 300-306.

[12] Jorge E, Silvio A, Jairo C. Determination of Cry toxin activity and identification of an aminopepidase $\mathrm{N}$ receptor-like gene in Asymmathetes vulcanorum (Coleoptera: Curculionidae), J. Invertebr. Pathol. 111 (2012) 94-98.

[13] Jurat-Fuentes JL, Adang MJ. Characterization of a Cry1Ac-receptor alkaline phosphatase in susceptible and resistant Heliothis virescens larvae, Eur. J. Biochem. 271 (2004) 3127-3135.

[14]Fernández LE, Aimanova KG Gill SS, et al. A GPI-anchored alkaline phosphatase is a functional midgut receptor of Cry11Aa toxin in Aedes aegypti larvae, Biochem. J. 394 (2006) 77-84.

[15] Tanaka S, Miyamoto K, Noda $H$, et al. Single amino acid insertions in extracellular loop 2 of Bombyx mori ABCC2 disrupt its receptor function for Bacillus thuringiensis Cry1Ab and Cry1 Ac but not Cry1Aa toxins, Peptides. 78 (2016) 99-108.

[16] Yuan Xiangdong, Zhang Wanna, Zhao Man, et al. Cloning, expression and functional analysis of alkaline phosphatase 2 (alp2) in the midgut of beet armyworm Spodoptera exigua (Hübner) (Lepidoptera: Noctuidae), J. Plant. Protection. 44 (2017) 8-15. In Chinese

[17] Jiménez AI, Reyes EZ, Cancino-Rodezno A, et al. Aedes aegypti alkaline phosphatase ALP1 is a functional receptor of Bacillus thuringiensis Cry4Ba and Cry11Aa toxins, Insect. Biochem. Mol. Biol. 42 (2012) 683-689.

[18]Dechklar M, Tiewsiri K, Angsuthanasombat C, et al. Functional expression in insect cells of glycosylphosphatidylinositol-linked alkaline phosphatase from Aedes aegypt larval midgut: a Bacillus thuringiensis Cry4Ba toxin receptor, Insect. Biochem. Mol. Biol. 41 (2011) 159-166. 
[19] Arenas I, Bravo A, Soberón M, et al. Role of alkaline phosphatase from Manduca sexta in the mechanism of action of Bacillus thuringiensis Cry1 Ab toxin, J. Biol. Chem. 285 (2010) 12497-503. 